\title{
Vibrations of Three-Layered Cylindrical Shells with FGM Middle Layer Resting on Winkler and Pasternak Foundations
}

\author{
Abdul Ghafar Shah, ${ }^{1}$ Aalia Ali, ${ }^{2}$ Muhmmad Nawaz Naeem, ${ }^{2}$ and Shahid Hussain Arshad ${ }^{3}$ \\ ${ }^{1}$ Government Post Graduate College Jhang, Jhang 35200, Punjab, Pakistan \\ ${ }^{2}$ G. C. University Faisalabad, Faisalabad 38000, Punjab, Pakistan \\ ${ }^{3}$ Department of Civil Engineering, Iqra National University Peshawar, Peshawar 25000, Pakistan
}

Correspondence should be addressed to Abdul Ghafar Shah, aghafarshah@yahoo.com

Received 28 April 2012; Revised 31 July 2012; Accepted 15 August 2012

Academic Editor: Marc Asselineau

Copyright (C) 2012 Abdul Ghafar Shah et al. This is an open access article distributed under the Creative Commons Attribution License, which permits unrestricted use, distribution, and reproduction in any medium, provided the original work is properly cited.

Vibrations of a cylindrical shell composed of three layers of different materials resting on elastic foundations are studied out. This configuration is formed by three layers of material in thickness direction where the inner and outer layers are of isotropic materials and the middle is of functionally graded material. Love shell dynamical equations are considered to describe the vibration problem. The expressions for moduli of the Winkler and Pasternak foundations are combined with the shell dynamical equations. The wave propagation approach is used to solve the present shell problem. A number of comparisons of numerical results are performed to check the validity and accuracy of the present approach.

\section{Introduction}

The circular cylindrical shells have been found in various engineering applications ranging from large civil and mechanical structures to small electrical components for many years. Vibrations of cylindrical shells are the most wide studied area of research because of their simple geometrical shapes. First of all, Love [1] presented the linear thin shell theory established on the Kirchhoff's hypothesis for plates. All other shell theories have been built on this theory by modifying some physical terms involving therein. Numerical solutions of shell vibration problem started to come out in the thirties of the twentieth century and were presented by Flügge [2]. Sharma [3,4] gave an analysis of thin circular cylinders. Sharma approximated the axial model dependence by beam functions and they used Rayleigh-Ritz technique to solve the shell problem. Paliwal et al. [5] investigated the vibrations of a thin circular cylindrical shell attached with elastic foundations. Loy et al. [6] investigated the vibrations of functionally graded material cylindrical shells, made up of FG material composed of stainless steel and nickel. The purpose of work was to examine natural frequencies, influence of the constituent volume fractions, and effects of configurations of constituent materials on their frequencies. Pardhan et al. [7] studied the vibrations of functionally graded material (FGM) cylindrical shell structured from stainless steel and zirconia. Zhang et al. [8] studied the vibration frequencies of cylindrical shells with fluid-filled. They used wave propagation approach and they compared the uncoupled frequencies with available results in the literature. Najafizadeh and Isvandzibaei [9] studied the vibrations of thin-walled cylindrical shells with ring supports composed of functionally graded material comprised of stainless steel and nickel. Arshad et al. [10] derived the frequency equation in the form of eigenvalue problem by employing Rayleigh-Ritz method. Love's thin shell theory was used for strain-displacement and curvaturedisplacement relation. They studied the natural frequency for simply supported boundary conditions and compared the results with those mentioned in the literature to check the validity of the approach. Iqbal et al. [11] applied wave propagation approach to analyze vibrations of functionally graded material circular cylindrical shells. They compared their results for the validity and accuracy by the results mentioned in the literature and found an excellent agreement between two sets of results. Arshad et al. [12] analyzed 
an associative study for natural frequencies of two-layered cylindrical shells that was presented with one layer made up of functionally graded material and the other layer of isotropic material. Strain-displacement and curvaturedisplacement relationships were derived by using Love's first shell theory. Shah et al. [13] presented an analysis on the vibrations of FG cylindrical shells founded on the Winkler and Pasternak foundations. The shell equations of motion were rearranged by introducing the moduli of the Winkler and Pasternak foundations. Shah et al. [14] studied the effects of volume fraction law on the vibration frequencies of thin FG cylindrical shells. Material parameters in shell radial direction were ranked according to the exponential law. Natural frequencies of the shell were dependent on the constituent volume fractions. The consequences were compared with those available in the literature for the validity of the presented approach. Arshad et al. [15] studied an analysis on vibrations of bilayered cylindrical shell made of two layers which were functionally graded. The thickness of the shell layers was considered to be equal and constant. Naeem et al. [16] studied the vibration frequency characteristics of functionally graded cylindrical shells using the generalized differential quadrature method. Shen et al. [17] studied the postbuckling response of a functionally graded cylindrical shell of finite length surrounded by an elastic medium and subjected to internal pressure in thermal environments. Sofiyev and Avear [18] investigated the stability of cylindrical shells that were composed of ceramic, FGM, and metal layers subjected to axial load and rested on Winkler-Pasternak foundations. Sofiyev et al. [19] analyzed the stability of functionally graded cylindrical shells subjected to combined loads with different edge conditions and resting on elastic foundations.

\section{Shell Problem}

A cylindrical shell of the thin wall is considered here as shown in Figure 1, with the geometrical parameters: length $L$, thickness $h$, and mean radius $R$. The orthogonal coordinate system $(x, \varphi, z)$ is taken to be at the surface of the shell where $x, \varphi$, and $z$ represent the axial, circumferential, and radial coordinates, respectively. Young's modulus E, the Poisson ratio $\nu$, and the mass density $\rho$ are the shell material parameters. The axial, circumferential, and radial displacement deformations are denoted by $u(x, \varphi, t), v(x$, $\varphi, t)$, and $w(x, \varphi, t)$, respectively, with regard to the shell middle surface.

For a cylindrical shell, plane stress condition can be assumed. From two-dimensional Hook's law, the constitutive relations for a thin cylindrical shell are stated mathematically as

$$
\{\sigma\}=[Q]\{e\},
$$

where $\{\sigma\}$ stands for the stress vector, $\{e\}$ corresponds to the strain vector, and $[Q]$ denotes the reduced stiffness matrix.
These stress and strain column vectors are defined as

$$
\begin{aligned}
& \{\sigma\}^{T}=\left\{\sigma_{x}, \sigma_{\varphi}, \sigma_{x \varphi}\right\}, \\
& \{e\}^{T}=\left\{e_{x}, e_{\varphi}, e_{x \varphi}\right\},
\end{aligned}
$$

where $\sigma_{x}$ and $\sigma_{\varphi}$ are linear stresses in the $x$ and $\varphi$-directions, and $\sigma_{x \varphi}$ is the shear stress in the $x \varphi$-plane. $e_{x}$ and $e_{\varphi}$ are strain in the directions $x$ and $\varphi$, and $e_{x \varphi}$ the shear strain in the $x \varphi$ plane. The reduced stiffness matrix is written as for the plane stress condition as

$$
[Q]=\left[\begin{array}{ccc}
Q_{11} & Q_{12} & 0 \\
Q_{12} & Q_{22} & 0 \\
0 & 0 & Q_{66}
\end{array}\right] .
$$

So the relation (1) can be expressed as

$$
\left\{\begin{array}{c}
\sigma_{x} \\
\sigma_{\varphi} \\
\sigma_{x \varphi}
\end{array}\right\}=\left[\begin{array}{ccc}
Q_{11} & Q_{12} & 0 \\
Q_{12} & Q_{22} & 0 \\
0 & 0 & Q_{66}
\end{array}\right]\left\{\begin{array}{c}
e_{x} \\
e_{\varphi} \\
e_{x \varphi}
\end{array}\right\} .
$$

The reduced stiffness $Q_{i j}(i, j=1,2$, and 6) for isotropic materials is defined as

$$
\begin{gathered}
Q_{11}=Q_{22}=\frac{E}{1-v^{2}}, \\
Q_{12}=\frac{v E}{1-v^{2}}, \\
Q_{66}=\frac{E}{2(1+v)} .
\end{gathered}
$$

The strain vector $\{e\}$ in its component form is defined as

$$
\begin{aligned}
& e_{x}=e_{1}+z \kappa_{1}, \\
& e_{\varphi}=e_{2}+z \kappa_{2}, \\
& e_{x \varphi}=\gamma+2 z \tau,
\end{aligned}
$$

where $e_{1}, e_{2}$, and $\gamma$ denote the reference surface strains. $\kappa_{1}$, $\kappa_{2}$, and $\tau$ represent the surface curvatures.

From the relations (4), (6a), (6b), and (6c), the stress vector $\{\sigma\}$ in its component form is defined as

$$
\begin{gathered}
\sigma_{x}=\left(e_{1}+z \kappa_{1}\right) Q_{11}+\left(e_{2}+z \kappa_{2}\right) Q_{12} \\
\sigma_{\varphi}=\left(e_{1}+z \kappa_{1}\right) Q_{12}+\left(e_{2}+z \kappa_{2}\right) Q_{22}, \\
\sigma_{x \varphi}=(\gamma+2 z \tau) Q_{66} .
\end{gathered}
$$

The force resultants and moment resultants for a cylindrical shell are written in the integral forms, respectively, as

$$
\begin{gathered}
\left\{N_{x}, N_{\varphi}, N_{x \varphi}\right\}=\int_{-h / 2}^{h / 2}\left\{\sigma_{x}, \sigma_{\varphi}, \sigma_{x \varphi}\right\} d z, \\
\left\{M_{x}, M_{\varphi}, M_{x \varphi}\right\}=\int_{-h / 2}^{h / 2}\left\{\sigma_{x}, \sigma_{\varphi}, \sigma_{x \varphi}\right\} z d z,
\end{gathered}
$$




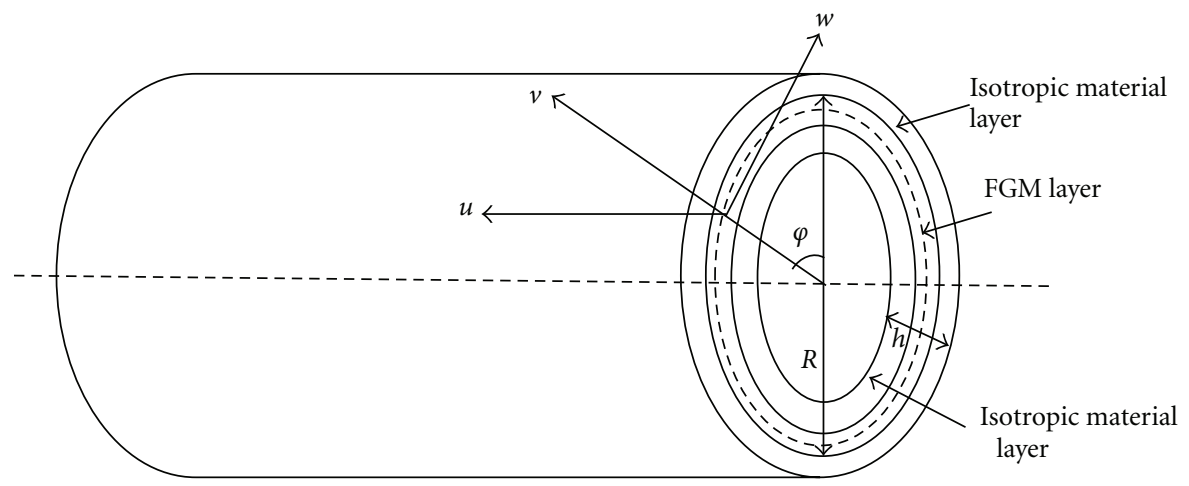

FIgURE 1: Geometry of three-layered cylindrical shell.

where $N_{x}, N_{\varphi}$, and $N_{x \varphi}$ are force components in axial, circumferential, and shear directions. $M_{x}, M_{\varphi}$, and $M_{x \varphi}$ are moment components in axial, circumferential, and shear directions.

The relations (6a), (6b), (6c), (8), and (9) imply that

$$
\{N\}=[K]\{\epsilon\},
$$

where $\{N\}$ and $\{\epsilon\}$ are defined as

$$
\begin{gathered}
\{N\}^{T}=\left\{N_{x}, N_{\varphi}, N_{x \varphi}, M_{x}, M_{\varphi}, M_{x \varphi}\right\}, \\
\{\epsilon\}^{T}=\left\{e_{1}, e_{2}, \gamma, \kappa_{1}, \kappa_{2}, 2 \tau\right\},
\end{gathered}
$$

and $[K]$ is defined as

$$
[K]=\left[\begin{array}{ll}
A & B \\
B & D
\end{array}\right]
$$

where $A, B$, and $D$ are submatrices of extensional, coupling, and boundary stiffness and are given as

$$
\begin{gathered}
{[A]=\left[\begin{array}{ccc}
A_{11} & A_{12} & 0 \\
A_{12} & A_{22} & 0 \\
0 & 0 & A_{66}
\end{array}\right], \quad[B]=\left[\begin{array}{ccc}
B_{11} & B_{12} & 0 \\
B_{12} & B_{22} & 0 \\
0 & 0 & B_{66}
\end{array}\right],} \\
{[D]=\left[\begin{array}{ccc}
D_{11} & D_{12} & 0 \\
D_{12} & D_{22} & 0 \\
0 & 0 & D_{66}
\end{array}\right] .}
\end{gathered}
$$

From $[A],[B]$, and $[C]$, a matrix $[K]$ can be written as

$$
[K]=\left[\begin{array}{cccccc}
A_{11} & A_{12} & 0 & B_{11} & B_{12} & 0 \\
A_{12} & A_{22} & 0 & B_{12} & B_{22} & 0 \\
0 & 0 & A_{66} & 0 & 0 & B_{66} \\
B_{11} & B_{12} & 0 & D_{11} & D_{12} & 0 \\
B_{12} & B_{22} & 0 & D_{12} & D_{22} & 0 \\
0 & 0 & B_{66} & 0 & 0 & D_{66}
\end{array}\right]
$$

where $A_{i j}, B_{i j}$, and $D_{i j}(i, j=1,2$, and 6) stand for extensional, coupling, and bending stiffness, respectively, and are defined as

$$
\begin{aligned}
A_{i j} & =\int_{-h / 2}^{h / 2} Q_{i j} d z, \\
B_{i j} & =\int_{-h / 2}^{h / 2} Q_{i j} z d z, \\
D_{i j} & =\int_{-h / 2}^{h / 2} Q_{i j} z^{2} d z .
\end{aligned}
$$

By using the relations (11)-(15), the expression (10) takes the following form:

$$
\left[\begin{array}{c}
N_{x} \\
N_{\varphi} \\
N_{x \varphi} \\
M_{x} \\
M_{\varphi} \\
M_{x \varphi}
\end{array}\right]=\left[\begin{array}{cccccc}
A_{11} & A_{12} & 0 & B_{11} & B_{12} & 0 \\
A_{12} & A_{22} & 0 & B_{12} & B_{22} & 0 \\
0 & 0 & A_{66} & 0 & 0 & B_{66} \\
B_{11} & B_{12} & 0 & D_{11} & D_{12} & 0 \\
B_{12} & B_{22} & 0 & D_{12} & D_{22} & 0 \\
0 & 0 & B_{66} & 0 & 0 & D_{66}
\end{array}\right]\left[\begin{array}{c}
e_{1} \\
e_{2} \\
\gamma \\
\kappa_{1} \\
\kappa_{2} \\
2 \tau
\end{array}\right] .
$$

The (coupling stiffness) $B_{i j}$ 's are vanished for isotropic cylindrical shells and are nonzero in case of FGM cylindrical shells.

For a thin-walled cylindrical shell that performs vibration, the formula for its strain energy denoted by $U$ is written as

$$
U=\frac{1}{2} \int_{0}^{L} \int_{0}^{2 \pi}\{\epsilon\}^{T}[K]\{\epsilon\} R d \varphi d x .
$$

By substitution of $\{\epsilon\}^{T},[K]$, and $\{\epsilon\}$ from (12) and (15) in the above expression, the new expression of the shell strain energy is written as

$$
\begin{aligned}
U=\frac{1}{2} \int_{0}^{L} \int_{0}^{2 \pi} & \left\{A_{11} e_{1}^{2}+A_{22} e_{2}^{2}+2 A_{12} e_{1} e_{2}+A_{66} \gamma^{2}\right. \\
& +2 B_{11} e_{1} \kappa_{1}+2 B_{12} e_{1} \kappa_{2}+2 B_{12} e_{2} \kappa_{1}+2 B_{22} e_{2} \kappa_{2} \\
& +4 B_{66} \gamma \tau+D_{11} \kappa_{1}^{2}+D_{22} \kappa_{2}^{2} \\
& \left.+2 D_{12} \kappa_{1} \kappa_{2}+4 D_{66} \tau^{2}\right\} d \varphi d x
\end{aligned}
$$


Also the kinetic energy of the cylindrical shell, represented by $T$, is expressed as

$$
T=\int_{0}^{L} \int_{0}^{2 \pi} \rho_{t}\left[\left(\frac{\partial u}{\partial t}\right)^{2}+\left(\frac{\partial v}{\partial t}\right)^{2}+\left(\frac{\partial w}{\partial t}\right)^{2}\right] R d \varphi d x
$$

where $t$ symbolizes the time variable and $\rho_{t}$ denotes the mass density per unit length and is defined as

$$
\rho_{t}=\int_{-h / 2}^{h / 2} \rho d z
$$

The present problem is performed by the Love's [1] first order thin shell theory and the analysis of expressions straindisplacement and curvature-displacement relationships are taken from this theory and are written as

$$
\begin{gathered}
\left\{e_{1}, e_{2}, \gamma\right\}=\left\{\frac{\partial u}{\partial x}, \frac{1}{R}\left(\frac{\partial v}{\partial \varphi}+w\right),\left(\frac{\partial v}{\partial x}+\frac{1}{R} \frac{\partial u}{\partial \varphi}\right)\right\} \\
\left\{\kappa_{1}, \kappa_{2}, \tau\right\}= \\
\left\{-\frac{\partial^{2} w}{\partial x^{2}},-\frac{1}{R^{2}}\left(\frac{\partial^{2} w}{\partial \varphi^{2}}-\frac{\partial v}{\partial \varphi}\right),-\frac{1}{R}\left(\frac{\partial^{2} w}{\partial x \partial \varphi}-\frac{\partial v}{\partial x}\right)\right\} .
\end{gathered}
$$

In a cylindrical shell executing vibration. The expressions for surface strains $e_{1}, e_{2}$, and $\gamma$ and the curvatures $\kappa_{1}, \kappa_{2}$, and $\tau$ from the relations (22), respectively, are substituted into (19), and the expression for strain energy is obtained as

$$
\begin{aligned}
U=\frac{1}{2} \int_{0}^{L} \int_{0}^{2 \pi}\{ & A_{11}\left(\frac{\partial u}{\partial x}\right)^{2}+\frac{A_{22}}{R^{2}}\left(\frac{\partial v}{\partial \varphi}+w\right)^{2}+2 \frac{A_{12}}{R}\left(\frac{\partial u}{\partial x}\right) \\
& \times\left(\frac{\partial v}{\partial \varphi}+w\right)+A_{66}\left(\frac{\partial v}{\partial x}+\frac{1}{R} \frac{\partial u}{\partial \varphi}\right)^{2}-2 B_{11} \\
& \times\left(\frac{\partial u}{\partial x}\right)\left(\frac{\partial^{2} w}{\partial x^{2}}\right)-2 \frac{B_{12}}{R^{2}}\left(\frac{\partial u}{\partial x}\right)\left(\frac{\partial^{2} w}{\partial x \partial \varphi}-\frac{\partial v}{\partial x}\right) \\
& -2 \frac{B_{12}}{R}\left(\frac{\partial v}{\partial \varphi}+w\right)\left(\frac{\partial^{2} w}{\partial x^{2}}\right)-2 \frac{B_{22}}{R^{3}} \\
\times & \left(\frac{\partial v}{\partial \varphi}+w\right)\left(\frac{\partial^{2} w}{\partial \varphi^{2}}-\frac{\partial v}{\partial \varphi}\right)-8 \frac{B_{66}}{R} \\
\times & \left(\frac{\partial v}{\partial x}+\frac{1}{R} \frac{\partial u}{\partial \varphi}\right)\left(\frac{\partial^{2} w}{\partial x \partial \varphi}-\frac{\partial v}{\partial x}\right)^{2} \\
& +D_{11}\left(\frac{\partial^{2} w}{\partial x^{2}}\right)^{2}+\frac{D_{22}}{R^{4}}\left(\frac{\partial^{2} w}{\partial \varphi^{2}}-\frac{\partial v}{\partial \varphi}\right)^{2} \\
& +2 \frac{D_{12}}{R^{2}}\left(\frac{\partial^{2} w}{\partial x^{2}}\right)\left(\frac{\partial^{2} w}{\partial \varphi^{2}}-\frac{\partial v}{\partial \varphi}\right) \\
& \left.\left.+\frac{\partial v}{\partial x}\right)^{2}\right\}^{2} d \varphi d x
\end{aligned}
$$

The Lagrangian energy functional represented by $\Pi$ for a circular cylindrical shell is defined by differentiating its strain and kinetic energies as

$$
\Pi=T-U .
$$

Hamilton's principle is employed to the Lagrangian energy functional $\Pi$, and introducing the Winkler and Pasternak foundations $\left(K w-G \nabla^{2} w\right)$ term in the $z$-direction is inducted and appended with the equations of motion to derive the shell motion equation written in a differential operator form as

$$
\begin{gathered}
L_{11} u+L_{12} v+L_{13} w=\rho_{t} \frac{\partial^{2} u}{\partial t^{2}}, \\
L_{21} u+L_{22} v+L_{23} w=\rho_{t} \frac{\partial^{2} v}{\partial t^{2}}, \\
L_{31} u+L_{32} v+L_{33} w=\rho_{t} \frac{\partial^{2} u}{\partial t^{2}}+K w-G \nabla^{2} w,
\end{gathered}
$$

where $L_{i j}(i, j=1,2,3)$ state the differential operators with regard to $x$ and $\varphi$ and are given in Appendix A, where $G$ stands for Pasternak elastic foundation and $K$ for the Winkler foundation modulus. The expression for the differential operator $\nabla^{2}$ is

$$
\nabla^{2}=\frac{\partial^{2}}{\partial x^{2}}+\frac{1}{R^{2}} \frac{\partial^{2}}{\partial \varphi^{2}} .
$$

The Pasternak model reduces to the Winkler model when $G$ vanishes.

Using the expressions for the differential operators $L_{i j}(i, j=1,2,3)$, the shells dynamical equations are written as

$$
\begin{aligned}
& e_{11} \frac{\partial^{2} u}{\partial x^{2}}+e_{12} \frac{\partial^{2} u}{\partial \varphi^{2}}+e_{13} \frac{\partial^{2} v}{\partial x \partial \varphi}+e_{14} \frac{\partial w}{\partial x}+e_{15} \frac{\partial^{3} w}{\partial x^{3}}+e_{16} \frac{\partial^{3} w}{\partial x \partial \varphi^{2}} \\
& =\rho_{t} \frac{\partial^{2} u}{\partial t^{2}}, \\
& e_{21} \frac{\partial^{2} u}{\partial x \partial \varphi}+e_{22} \frac{\partial^{2} v}{\partial x^{2}}+e_{23} \frac{\partial^{2} v}{\partial \varphi^{2}}+e_{24} \frac{\partial^{3} w}{\partial x^{2} \partial \varphi}+e_{25} \frac{\partial w}{\partial \varphi}+e_{26} \frac{\partial^{3} w}{\partial \varphi^{3}} \\
& =\rho_{t} \frac{\partial^{2} v}{\partial t^{2}},
\end{aligned}
$$

$$
\begin{gathered}
e_{31} \frac{\partial^{3} u}{\partial x^{3}}+e_{32} \frac{\partial u}{\partial x}+e_{33} \frac{\partial^{3} u}{\partial x \partial \varphi^{2}}+e_{34} \frac{\partial^{3} v}{\partial x^{2} \partial \varphi}+e_{35} \frac{\partial^{3} v}{\partial \varphi^{3}}+e_{36} \frac{\partial v}{\partial \varphi} \\
+e_{37} \frac{\partial^{4} w}{\partial x^{4}}+e_{38} \frac{\partial^{4} w}{\partial x^{2} \partial \varphi^{2}}+e_{39} \frac{\partial^{4} w}{\partial \varphi^{4}}+e_{310} \frac{\partial^{2} w}{\partial x^{2}}+e_{311} \frac{\partial^{2} w}{\partial \varphi^{2}}+e_{312} w \\
=\rho_{t} \frac{\partial^{2} w}{\partial t^{2}}+K w-G\left(\frac{\partial^{2} w}{\partial x^{2}}+e_{313} \frac{\partial^{2} w}{\partial \varphi^{2}}\right)
\end{gathered}
$$

where $e_{i j}^{\prime} s$ are defined in Appendix B. 


\section{Methodology}

The following modal displacement shape functions are adopted to separate the time and space variables:

$$
\begin{gathered}
u(x, \varphi, t)=A \cos (n \varphi) e^{i\left(\omega t-k_{m} x\right)}, \\
v(x, \varphi, t)=B \sin (n \varphi) e^{i\left(\omega t-k_{m} x\right)}, \\
w(x, \varphi, t)=C \cos (n \varphi) e^{i\left(\omega t-k_{m} x\right)},
\end{gathered}
$$

in the longitudinal, circumferential, and transverse directions, respectively. The constants $A, B$, and $C$ are the amplitudes of vibrations in the $x, \varphi$, and $z$ directions, respectively, $n$ is the number of circumferential waves, and $k_{m}$ stands for axial wave number that is associated with a boundary condition is axial wave number given for four types of boundary conditions in Table 1 .

These axial wave numbers $k_{m}$ are selected to satisfy boundary conditions at both edges of a cylindrical shell. $\omega$ denotes the natural angular frequency for the cylindrical shell.

\section{Derivation of Frequency Equation}

Making substitution for the displacement functions $u, v$, and $w$ from the expressions (28) in system of (27a), (27b), and $(27 \mathrm{c})$, the shell algebraic equations are written as:

$$
\begin{gathered}
c_{11} A+c_{12} B+c_{13} C=\omega^{2} \rho_{t} A, \\
c_{21} A+c_{22} B+c_{23} C=\omega^{2} \rho_{t} B \\
c_{31} A+c_{32} B+c_{33} C+K w+G\left(k_{m}^{2}+e_{313} n^{2}\right)=\omega^{2} \rho_{t} C
\end{gathered}
$$

where $c_{i j}^{\prime} s(i, j=1,2,3)$ are given in Appendix C.

After the arrangement of terms, the algebraic simultaneous equations (29a)-(29c) are written in matrix notation as

$$
\left[\begin{array}{ccc}
c_{11} & c_{12} & c_{13} \\
-c_{21} & c_{22} & c_{23} \\
-c_{13} & c_{23} & c_{33}
\end{array}\right]\left[\begin{array}{l}
A \\
B \\
C
\end{array}\right]=\omega^{2} \rho_{t}\left[\begin{array}{lll}
1 & 0 & 0 \\
0 & 1 & 0 \\
0 & 0 & 1
\end{array}\right]\left[\begin{array}{l}
A \\
B \\
C
\end{array}\right] .
$$

For forming the shell frequency equation, the determinant of the matrix coefficients is vanished for nontrivial eigenvalue of the shell frequency. Either the eigenvalue problem obtained above is solved using computer program or the determinant is expanded and the frequencies equation is achieved in the form of polynomial equation involving $\omega^{2}$.

\section{Results and Discussion}

Numerical results for simply supported-simply supported cylindrical shell are calculated and compared with those found in the open literature in order to examine validity, efficiency, and accuracy of wave propagation approach. In Table 2, the nondimensional frequency parameters $\Omega=$ $\omega R \sqrt{\left(1-v^{2}\right)} \rho / E$ for an isotropic cylindrical shell are compared with those ones obtained by Naeem and Sharma [20].
The simply supported boundary conditions are described on shell ends. In this comparison, the shell parameters: length $L=6 m$, thickness $h=0.002 m$, and $R=1 m$, are taken, respectively. The axial mode is assumed to be $m=1$, and the circumferential wave numbers $n$ are chosen from 1-10. Material properties of the shell are mass density $(\rho)$, Poisson's ratio $(\nu)$, and Young's modulus $(E)$ and their values are given as $\rho=7850 \mathrm{~kg} / \mathrm{m}^{3}, \nu=0.3$, and $E=2.1 \times 10^{11} \mathrm{~N} / \mathrm{m}^{2}$.

In Table 3 , the frequency parameters $\Omega$ for a cylindrical shell with simply supported boundary conditions are compared with those results determined by Zhang et al. [8]. The geometrical parameters are assumed to be as $L=20 \mathrm{~m}$, $h=0.002 \mathrm{~m}$, and $R=1 \mathrm{~m}$. For the parameters $L / R$, a value of $m=1$ is used and $n$ are selected $0-4$ in the comparison. The material properties of the shell are given as $\rho=7850 \mathrm{~kg} / \mathrm{m}^{3}$, $\nu=0.3$, and $E=2.1 \times 10^{11} \mathrm{~N} / \mathrm{m}^{2}$.

\section{Three-Layered Cylindrical Shells}

A number of results for the proposed three-layered functionally graded cylindrical shells are determined for various sets of material and geometrical parameters. The inner and outer layers of the shell are comprised of isotropic material, whereas the middle layer is assumed to be functionally graded as shown in Figure 1. In general, vibration characteristics are most influenced by Young's modulus. In this study, the Poisson's ratio is presumed to be constant for functionally graded materials, whereas the Young's modulus depends on intrinsic thickness variable $(z)$ as well as the Young's modulus of constituent materials forming functionally graded layers. Here two configurations of a cylindrical shell are considered to suggest with regard to the shell layer thickness. In the first configuration, the thickness of each layer is supposed to be of $h / 3$ while in the second configuration, the thickness of each of the inner and outer layers is of $h / 4$ and that of the middle layer is of $h / 2$.

The stiffness moduli $A_{i j}, B_{i j}$, and $D_{i j}$ are modified in according to the thickness of material layers when inner and outer layers are isotropic and middle, is functionally graded as

$$
\begin{aligned}
& A_{i j}=A_{i j}^{\mathrm{in}(\text { isotropic })}+A_{i j}^{m(\mathrm{FG})}+A_{i j}^{\text {out(isotropic) }}, \\
& B_{i j}=B_{i j}^{\text {in(Isotropic) }}+B_{i j}^{m(\mathrm{FG})}+B_{i j}^{\text {out(isotropic })}, \\
& D_{i j}=D_{i j}^{\text {in(isotropic) }}+D_{i j}^{m(\mathrm{FG})}+D_{i j}^{\text {out(isotropic })},
\end{aligned}
$$

where $i, j=1,2,6$ and in(isotropic), $m$ (FG), and out(isotropic) are associated with inner isotropic, middle functionally graded and outer isotropic layers of cylindrical shell, respectively. Here, by considering the constituent materials of stainless steel and aluminum for isotropic layers and also the FG layers which are structured from two kinds of materials, nickel and zirconia four types of shells are obtained and are listed Table 4.

Material properties of isotropic materials: steel and aluminum are given in Table 5, whereas the material properties 


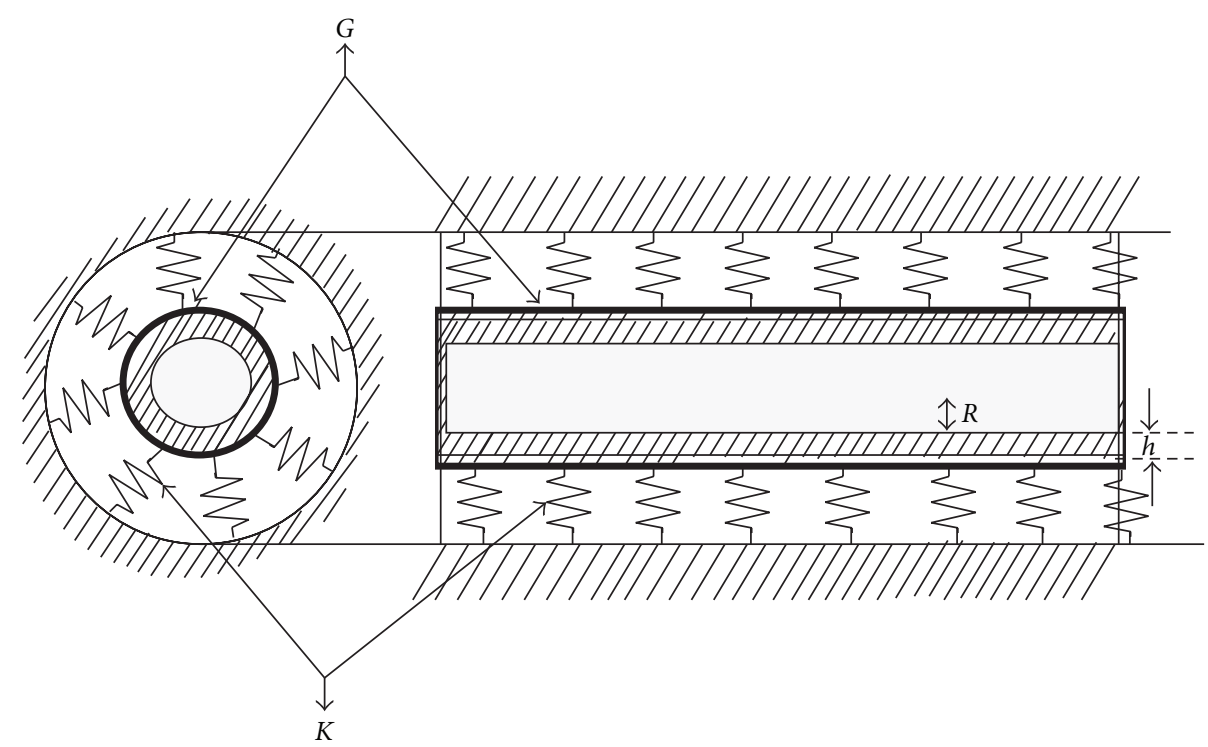

Figure 2: Cylindrical shell with elastic foundations.

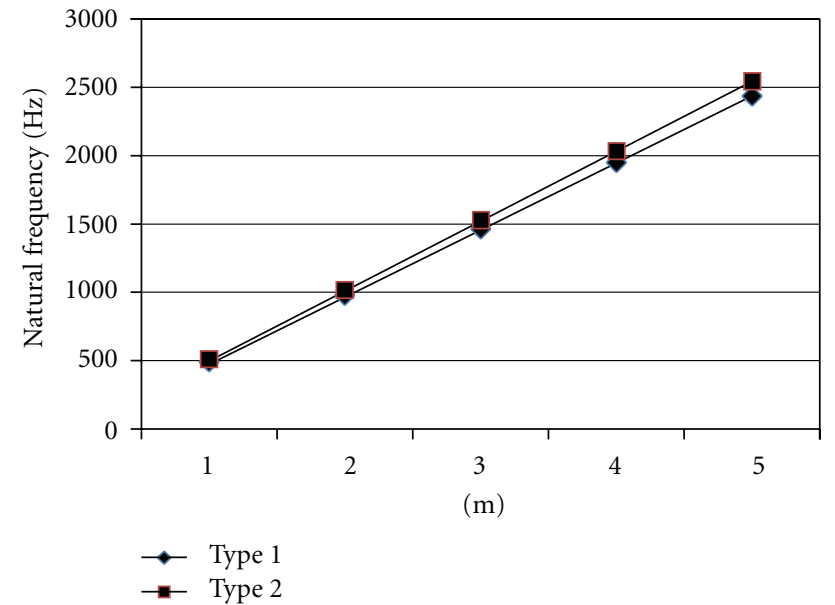

Figure 3: Variations of natural frequencies $(\mathrm{Hz})$ for three-layered SS-SS cylindrical shells $\left(G=1 \times 10^{9} \mathrm{~N} \cdot \mathrm{m}, K=1 \times 10^{9}\right)$.

TABLE 1

\begin{tabular}{lc}
\hline Boundary conditions & Wave numbers \\
\hline Simply supported-simply supported & $k_{m}=m \pi / L$ \\
Clamped-clamped & $k_{m}=(2 m+1) \pi / 2 L$ \\
Clamped-simply supported & $k_{m}=(4 m+1) \pi / 4 L$ \\
Clamped-free & $k_{m}=(2 m-1) \pi / 2 L$ \\
\hline
\end{tabular}

of the constituent materials forming functionally graded layers are listed in Table 6.

\section{Functionally Graded Materials}

The FGMs are much advanced materials and are used in engineering science and technology. Material properties of an FGM are the functions of the temperature and the volume
TABLE 2: Comparison of frequency parameter $\Omega=\omega R \sqrt{\left(1-\nu^{2}\right) \rho / E}$ for SS-SS cylindrical shell $(m=1, L=6 m, h=0.002 m, R=1 m$, $v=0.3)$.

\begin{tabular}{lccc}
\hline$n$ & Naeem and Sharma [20] & Present & Difference percentage \\
\hline 1 & 0.140641 & 0.140642 & 0.000 \\
2 & 0.054323 & 0.054324 & 0.002 \\
3 & 0.027074 & 0.027075 & 0.004 \\
4 & 0.017776 & 0.017767 & -0.051 \\
5 & 0.017088 & 0.017074 & -0.082 \\
6 & 0.021303 & 0.021304 & 0.005 \\
7 & 0.028089 & 0.028082 & -0.025 \\
8 & 0.036469 & 0.036470 & 0.003 \\
9 & 0.046174 & 0.046171 & -0.006 \\
10 & 0.057088 & 0.057088 & 0.000 \\
\hline
\end{tabular}

TABLE 3: Comparison of frequency parameter $\Omega=\omega R \sqrt{\left(1-v^{2}\right) \rho / E}$ for SS-SS cylindrical shell $(m=1, L / R=20, v=0.3)$.

\begin{tabular}{ccccc}
\hline$h / R$ & $n$ & Zhang et al. [8] & Present & Difference percentage \\
\hline & 0 & 0.0929586 & 0.0929296 & -0.0311967 \\
& 1 & 0.0161011 & 0.0161011 & 0.0000000 \\
0.002 & 2 & 0.0054532 & 0.0054533 & 0.0018338 \\
& 3 & 0.0050418 & 0.0050419 & 0.0019834 \\
& 4 & 0.0085340 & 0.0085344 & 0.0046871 \\
\hline
\end{tabular}

fractions. These properties of a constituent material are managed by a volume fraction. If $P_{i}$ represents a material property of the $i$ th constituent material of an FGM consisting of $k$ constituent materials, then the effective property $P$ of the FGM is written as

$$
P=\sum_{i=1}^{k} P_{i} V_{i},
$$


TABLE 4: Description of cylindrical shells.

\begin{tabular}{lccc}
\hline Types & Isotropic inner & FGM material middle & Isotropic outer \\
\hline Type 1 & Stainless steel & Zirconia-Nickel & Stainless steel \\
Type 2 & Stainless steel & Nickel-Zirconia & Stainless steel \\
Type 3 & Stainless steel & Zirconia-Nickel & Aluminum \\
Type 4 & Stainless steel & Nickel-Zirconia & Aluminum \\
\hline
\end{tabular}

TABLE 5: Material properties of isotropic materials.

\begin{tabular}{lccc}
\hline Isotropic & $E\left(\mathrm{~N} / \mathrm{m}^{2}\right)$ & Poisson ratio $(\nu)$ & Density $\rho\left(\mathrm{Kg} / \mathrm{m}^{3}\right)$ \\
\hline Stainless steel & $68.95 E+09$ & 0.315 & $2.7145 E+03$ \\
Aluminum & $2.1 E+11$ & 0.28 & $7.8 E+03$
\end{tabular}

TABLE 6: Material properties of Nickel and Zirconia.

\begin{tabular}{lccc}
\hline FGM & $E\left(\mathrm{~N} / \mathrm{m}^{2}\right)$ & Poisson ratio $(\nu)$ & Density $\rho\left(\mathrm{Kg} / \mathrm{m}^{3}\right)$ \\
\hline Nickel & $2.05098 E+11$ & 0.310 & 8900 \\
Zirconia & $1.68063 E+11$ & 0.298 & 5700 \\
\hline
\end{tabular}

TABLe 7: Variation of natural frequencies $(\mathrm{Hz})$ of a three-layered simply supported-simply supported cylindrical shell $(m=1, p=5$, $L=20 m, h=0.002 m, R=1 m)$.

\begin{tabular}{ccccc}
\hline$n$ & Type 1 & Type 2 & Type 3 & Type 4 \\
\hline 1 & 13.2769 & 13.8916 & 13.5193 & 13.9734 \\
2 & 4.4514 & 4.6301 & 4.5685 & 4.7054 \\
3 & 3.6084 & 3.8123 & 4.0553 & 4.2354 \\
4 & 5.7794 & 6.2181 & 6.7519 & 7.1280 \\
5 & 9.1613 & 9.9023 & 10.7583 & 11.3856 \\
\hline
\end{tabular}

TABLE 8: Variation of natural frequencies $(\mathrm{Hz})$ of a three-layered simply supported-simply supported cylindrical shell for two configurations with respect to thickness of shell layers $(m=1, p=5$, $L=20 m, h=0.002 m, R=1 m)$.

\begin{tabular}{cccc}
\hline$n$ & Type $1(h / 3)$ & Type $1(h / 4)$ & Difference percentage \\
\hline 1 & 13.2769 & 13.2145 & 0.4700 \\
2 & 4.4514 & 4.4410 & 0.2336 \\
3 & 3.6084 & 3.5684 & 1.1085 \\
4 & 5.7794 & 5.6616 & 2.0383 \\
5 & 9.1613 & 8.9530 & 2.2737 \\
\hline
\end{tabular}

where $V_{i}$ is the volume fraction of the $i$ th constituent material. Also, the sum of volume fractions of the constituent materials is equal to 1 , that is,

$$
\sum_{i=1}^{k} V_{i}=1
$$

The volume fraction depends on the thickness variable and is defined as

$$
V_{i}=\left(\frac{Z-R_{i}}{R_{o}-R_{i}}\right)^{N},
$$

TABLE 9: Variation of natural frequencies $(\mathrm{Hz})$ of a three-layered clamped-clamped cylindrical shell for two configurations with respect to thickness of shell layers $(m=1, p=5, L=20 m$, $h=0.002 m, R=1 m)$.

\begin{tabular}{cccc}
\hline$n$ & Type $1(h / 3)$ & Type $1(h / 4)$ & Difference percentage \\
\hline 1 & 28.7544 & 28.6163 & 0.4803 \\
2 & 9.6851 & 9.6514 & 0.3480 \\
3 & 5.4815 & 5.4589 & 0.4123 \\
4 & 6.2720 & 6.1775 & 1.5067 \\
5 & 9.3122 & 9.1194 & 2.0704 \\
\hline
\end{tabular}

TABLe 10: Variation of natural frequencies $(\mathrm{Hz})$ of a simply supported-simply supported three-layered cylindrical shell on elastic foundations $(m=1, p=1, L=0.41, R=0.3015, h=0.001$, $\left.v=0.3, G=1.5 \times 10^{7} \mathrm{~N} \cdot \mathrm{m}, K=2.5 \times 10^{7} \mathrm{~N} \cdot \mathrm{m}\right)$.

\begin{tabular}{ccccc}
\hline$n$ & Type 1 & Type 2 & Type 3 & Type 4 \\
\hline 1 & 8405.31 & 8395.60 & 7662.73 & 7675.80 \\
2 & 9318.72 & 9299.60 & 9044.04 & 9060.03 \\
3 & 11100.6 & 11074.7 & 11036.5 & 11055.3 \\
4 & 13322.2 & 13290.9 & 13335.4 & 13357.7 \\
5 & 15748.9 & 15712.0 & 15802.7 & 15828.9 \\
\hline
\end{tabular}

for a cylindrical shell. $R_{i}$ and $R_{o}$ denote inner and outer radii of the shell, respectively, and $z$ is the thickness variable in the radial direction. $N$ is known as the power law exponent. It is a nonnegative real number and lies between zero and infinity. For a cylindrical shell, the volume fraction is assumed as

$$
V_{i}=\left(\frac{Z+0.5 h}{h}\right)^{N},
$$

where $h$ is the shell uniform thickness. When the shell is considered to consist of two materials, the effective Young's modulus $E$, the poisson ratio $v$, and the mass density $\rho$ are given by

$$
\begin{aligned}
& E=\left(E_{1}-E_{2}\right)\left(\frac{Z+0.5 h}{h}\right)^{N}+E_{2}, \\
& v=\left(v_{1}-v_{2}\right)\left(\frac{Z+0.5 h}{h}\right)^{N}+v_{2}, \\
& \rho=\left(\rho_{1}-\rho_{2}\right)\left(\frac{Z+0.5 h}{h}\right)^{N}+\rho_{2} .
\end{aligned}
$$

\section{Frequency Analysis of Cylindrical Shells}

8.1. Variations of Natural Frequencies (Hz) against Circumferential Wave Number (n). In Table 7, natural frequencies $(\mathrm{Hz})$ of four types of cylindrical shells are given against the circumferential wave numbers $(n)$. The boundary conditions applied on the shell ends are simply supported. The shell parameter data is $m=1, L=20, h=0.002$, and $R=1$. For functionally graded layer, the power law exponent $(p)$ is 5 . The numerical results exhibit the well-known characteristic 
TABLE 11: Variations of natural frequencies (Hz) of SS-SS cylindrical shell for different values of $G$, where $K=2.5 \times 10^{7} \mathrm{~N} \cdot \mathrm{m}, h=0.001$, $m=1, R=1, L=0.41$.

\begin{tabular}{|c|c|c|c|c|c|}
\hline Shell type & $n$ & $G=1.5 \times 10^{7}$ & $G=2.5 \times 10^{7}$ & $G=3.5 \times 10^{7}$ & $G=4.5 \times 10^{7}$ \\
\hline \multirow{6}{*}{ Type 1} & 1 & 6527.31 & 6528.00 & 6528.86 & 6529.95 \\
\hline & 2 & 6690.30 & 6691.12 & 6692.14 & 6693.43 \\
\hline & 3 & 6953.44 & 6954.45 & 6955.71 & 6957.31 \\
\hline & 4 & 7305.79 & 7307.05 & 7308.60 & 7310.57 \\
\hline & 5 & 7734.99 & 7736.49 & 7738.35 & 7740.71 \\
\hline & 6 & 8228.81 & 8230.54 & 8232.68 & 8235.40 \\
\hline \multirow{6}{*}{ Type 2} & 1 & 6542.66 & 6543.38 & 6544.27 & 6545.40 \\
\hline & 2 & 6706.04 & 6706.88 & 6707.93 & 6709.26 \\
\hline & 3 & 6969.79 & 6970.83 & 6972.12 & 6973.75 \\
\hline & 4 & 7322.95 & 7324.23 & 7325.81 & 7327.82 \\
\hline & 5 & 7753.13 & 7754.66 & 7756.54 & 7758.93 \\
\hline & 6 & 8248.07 & 8249.82 & 8251.99 & 8254.73 \\
\hline \multirow{6}{*}{ Type 3} & 1 & 6580.75 & 6581.15 & 6581.61 & 6582.14 \\
\hline & 2 & 6745.0 & 6745.51 & 6746.07 & 6746.70 \\
\hline & 3 & 7010.27 & 7010.87 & 7011.56 & 7012.37 \\
\hline & 4 & 7365.46 & 7366.21 & 7367.08 & 7368.09 \\
\hline & 5 & 7798.15 & 7799.06 & 7800.11 & 7801.32 \\
\hline & 6 & 8296.01 & 8297.07 & 8298.29 & 8299.70 \\
\hline \multirow{6}{*}{ Type 4} & 1 & 6591.61 & 6592.02 & 6592.50 & 6593.05 \\
\hline & 2 & 6756.17 & 6756.66 & 6757.22 & 6757.88 \\
\hline & 3 & 7021.84 & 7022.45 & 7023.16 & 7023.98 \\
\hline & 4 & 7377.61 & 7378.38 & 7379.26 & 7380.28 \\
\hline & 5 & 7811.00 & 7811.92 & 7812.98 & 7814.21 \\
\hline & 6 & 8309.66 & 8310.73 & 8311.96 & 8313.38 \\
\hline
\end{tabular}

of shell vibration frequencies, that is, the value of the frequency first decreases achieves its lowest value and then it begins to increase with the circumferential wave modes $(n)$.

For Type 1 and 2, the outer and inner layers are of steel. This shows that the values of shell frequencies increase with interchanging the order of constituent materials forming the functionally graded middle layer. For Type 1, the inner and outer layer is of steel whereas in Type 3, the inner is of steel and the outer is of aluminum. The frequency increases with the interchange of inner and outer layers material whereas the configuration of functionally graded layer is the same. Also in Type 4, the inner and outer layers possess the same materials as in Type 3, where the materials of middle layer have been replaced by changing the order of zirconia and nickel. This shows that the frequency has been increased by changing the materials of functionally graded layer.

In Tables 8 and 9, variations of natural frequencies for Type 1 for two configurations with respect to thickness of layers are shown against circumferential wave numbers $(n)$. It is seen from Table 8 that by decreasing the thickness of the middle functionally graded layer, the frequencies diminish.

8.2. Frequency Analysis of Cylindrical Shells on Elastic Foundations. Figure 3 represents variations of natural frequencies $(\mathrm{Hz})$ for three-layered cylindrical shells for SS-SS edge conditions with elastic foundations, that is, $G=1 \times 10^{9}$,
$K=1 \times 10^{9} \mathrm{~N} \cdot \mathrm{m}$. The middle layer is functionally graded material. For both Types 1 and 2 of the cylindrical shells, the frequencies increase considerably by adding the elastic foundations, that is, Pasternak and Winkler models. Also, the values of natural frequencies increase gradually against circumferential wave modes $(n)$. The minimum frequency is associated with $n=1$. This shows that the shell vibration is similar to that of beam type for these boundary conditions.

In Table 10, natural frequencies $(\mathrm{Hz})$ for SS-SS threelayered cylindrical shells are listed for Type 1, 2, 3, and 4 cylindrical shells. The shells are based on the Pasternak and Winkler foundations as shown in Figure 2. The values of the shell frequencies show considerable increments by inducting the elastic moduli. Type 1 has the highest frequency, followed by Type 2, Type 4, and Type 3. This also exhibits the effects of material thickness of the layers forming cylindrical shell.

Table 11 displays the variations of natural frequencies (Hz) for four cylindrical shells: Type 1, Type 2, Type 3, and Type 4 for simply supported boundary conditions. The half axial wave mode number $(m)$ is assumed to be one. Here, $K=2.5 \times 10^{7} \mathrm{~N} \cdot \mathrm{m}$ and $G$ is varied. As $G$ is increased, the frequency increases gradually but slowly for the present four types of shells. Also for circumferential wave numbers $(n)$, the frequency increases as $\mathrm{n}$ is increased. The minimum frequency corresponds to the circumferential wave number $n=1$. The vibration becomes beam-type because the fundamental circumferential mode is 1 . 
TABLE 12: Variations of natural frequencies (Hz) of SS-SS cylindrical shell for different values of $K$, where $G=2.5 \times 10^{7} \mathrm{~N} \cdot \mathrm{m}, h=0.001$, $m=1, R=1, L=0.41$.

\begin{tabular}{|c|c|c|c|c|c|}
\hline Shell type & $n$ & $K=1.5 \times 10^{7}$ & $K=2.5 \times 10^{7}$ & $K=3.5 \times 10^{7}$ & $K=4.5 \times 10^{7}$ \\
\hline \multirow{6}{*}{ Type 1} & 1 & 6527.31 & 6527.33 & 6527.33 & 6527.35 \\
\hline & 2 & 6690.30 & 6690.32 & 6690.33 & 6690.34 \\
\hline & 3 & 6953.44 & 6953.45 & 6953.47 & 6953.48 \\
\hline & 4 & 7305.79 & 7305.81 & 7305.82 & 7305.84 \\
\hline & 5 & 7734.99 & 7735.01 & 7735.02 & 7735.04 \\
\hline & 6 & 8228.81 & 8228.82 & 8228.84 & 8228.86 \\
\hline \multirow{6}{*}{ Type 2} & 1 & 6542.66 & 6542.67 & 6542.68 & 6542.69 \\
\hline & 2 & 6706.04 & 6706.05 & 6706.06 & 6706.07 \\
\hline & 3 & 6969.79 & 6969.80 & 6969.814 & 6969.83 \\
\hline & 4 & 7322.95 & 7322.97 & 7322.98 & 7323.00 \\
\hline & 5 & 7753.13 & 7753.15 & 7753.16 & 7753.18 \\
\hline & 6 & 8248.07 & 8248.09 & 8248.12 & 8248.12 \\
\hline \multirow{6}{*}{ Type 3} & 1 & 6580.75 & 6580.75 & 6580.76 & 6580.77 \\
\hline & 2 & 6745.0 & 6745.04 & 6745.04 & 6745.05 \\
\hline & 3 & 7010.27 & 7010.27 & 7010.28 & 7011.0 \\
\hline & 4 & 7365.46 & 7365.47 & 7365.48 & 7365.49 \\
\hline & 5 & 7798.15 & 7798.16 & 7798.17 & 7798.18 \\
\hline & 6 & 8296.01 & 8296.02 & 8296.03 & 8296.04 \\
\hline \multirow{6}{*}{ Type 4} & 1 & 6591.61 & 6591.62 & 6591.63 & 6591.63 \\
\hline & 2 & 6756.17 & 6756.17 & 6756.18 & 6756.19 \\
\hline & 3 & 7021.84 & 7021.85 & 7021.87 & 7021.87 \\
\hline & 4 & 7377.61 & 7377.62 & 7377.63 & 7377.64 \\
\hline & 5 & 7811.00 & 7811.01 & 7811.02 & 7811.03 \\
\hline & 6 & 8309.66 & 8309.67 & 8309.68 & 8309.69 \\
\hline
\end{tabular}

In Table 12, the natural frequency variations $(\mathrm{Hz})$ for four cylindrical shells Type 1, Type 2, Type 3, and Type 4 are given against circumferential wave numbers $(n)$. The value of Pasternak modulus $(G)$ is maintained and fixed, that is, $G=2.5 \times 10^{7} \mathrm{~N} \cdot \mathrm{m}$ for the half axial wave mode $m=1$. The values of shell frequencies do not increase much with increasing value of $K$. The smallest frequency is associated with $n=1$, that is, the vibration is similar as that of a beam. Thus the effect of $K$ is negligible for keeping $G$ constant.

\section{Conclusion}

In this study, vibrations of three-layered cylindrical shells are investigated for various shell parameters. Three shell layers are designed by isotropic and functionally graded materials. In the configuration, outer and inner layers are of isotropic layers, whereas the middle layer is assumed to be of functionally graded layers. This forms a combination of isotropic and functionally graded layers. In a shell thickness direction, material composition of a functionally graded material is controlled by volume fraction law. Love shell dynamical equations are considered to describe the vibration problem. Pasternak and Winkler foundations are appended in the transverse direction. The boundary conditions applied on the shell ends are simply supported. Wave propagation approach is utilized to frame the shell frequency equation for a cylindrical shell in the eigenvalue form. The influences of the configurations of the cylindrical shells are analyzed with interchange of materials of shell layers. From this analysis, it is observed that the change of material nature of the shell functionally graded layer influences the shell frequencies. As the thickness of the middle layer is decreased, the vibration frequencies diminish prominently. For cylindrical shells of Types 1 and 2, the vibration frequencies increase considerably by adding the elastic foundations, that is, Pasternak and Winkler models. This analysis can be applied to extract vibration frequencies for the present cylindrical shell configurations for various boundary conditions.

\section{Appendices}

A.

$$
\begin{gathered}
L_{11}=A_{11} \frac{\partial^{2}}{\partial x^{2}}+\frac{A_{66}}{R^{2}} \frac{\partial^{2}}{\partial \varphi^{2}}, \\
L_{12}=\frac{\left(A_{12}+A_{66}\right)}{R} \frac{\partial^{2}}{\partial x \partial \varphi}+\frac{\left(B_{12}+2 B_{66}\right)}{R^{2}} \frac{\partial^{2}}{\partial x \partial \varphi}, \\
L_{13}=\frac{A_{12}}{R} \frac{\partial}{\partial x}-B_{11} \frac{\partial^{3}}{\partial x^{3}}-\frac{\left(B_{12}+2 B_{66}\right)}{R^{2}} \frac{\partial^{3}}{\partial x \partial \varphi^{2}}, \\
L_{21}=\frac{\left(A_{12}+A_{66}\right)}{R} \frac{\partial^{2}}{\partial x \partial \varphi}+\frac{\left(B_{12}+B_{66}\right)}{R^{2}} \frac{\partial^{2}}{\partial x \partial \varphi},
\end{gathered}
$$




$$
\begin{gathered}
L_{22}=\left(A_{66}+\frac{3 B_{66}}{R}+\frac{2 D_{66}}{R^{2}}\right) \frac{\partial^{2}}{\partial x^{2}} \\
+\left(\frac{A_{22}}{R^{2}}+\frac{2 B_{22}}{R^{3}}+\frac{D_{22}}{R^{4}}\right) \frac{\partial^{2}}{\partial \varphi^{2}}, \\
L_{23}=\left(\frac{A_{22}}{R^{2}}+\frac{B_{22}}{R^{3}}\right) \frac{\partial}{\partial \varphi}-\left(\frac{B_{22}}{R^{3}}+\frac{D_{22}}{R^{4}}\right) \frac{\partial^{3}}{\partial \varphi^{3}} \\
-\left(\frac{B_{12}+2 B_{66}}{R}+\frac{D_{12}+2 D_{66}}{R^{2}}\right) \frac{\partial^{3}}{\partial x^{2} \partial \varphi}, \\
L_{31}=-\frac{A_{12}}{R} \frac{\partial}{\partial x}+B_{11} \frac{\partial^{3}}{\partial x^{3}}+\frac{\left(B_{12}+2 B_{66}\right)}{R^{2}} \frac{\partial^{3}}{\partial x \partial \varphi^{2}}, \\
L_{32}=-\left(\frac{A_{22}}{R^{2}}+\frac{B_{22}}{R^{3}}\right) \frac{\partial}{\partial \varphi}+\left(\frac{B_{22}}{R^{3}}+\frac{D_{22}}{R^{4}}\right) \frac{\partial^{3}}{\partial \varphi^{3}} \\
+\left(\frac{B_{12}+2 B_{66}}{R}+\frac{D_{12}+4 D_{66}}{R^{2}}\right) \frac{\partial^{3}}{\partial x^{2} \partial \varphi} \\
L_{33}=-\frac{A_{22}}{R^{2}}+\frac{2 B_{12}}{R} \frac{\partial^{2}}{\partial x^{2}}+\frac{2 B_{22}}{R^{3}} \frac{\partial^{2}}{\partial \varphi^{2}}-2 D_{11} \frac{\partial^{4}}{\partial x^{4}} \\
\partial x^{2} \partial \varphi^{2}-\frac{D_{22}}{R^{4}} \frac{\partial^{4}}{\partial \varphi^{4}} .
\end{gathered}
$$

B.

$$
\begin{gathered}
e_{11}=A_{11}, \quad e_{12}=\frac{A_{66}}{R^{2}}, \\
e_{13}=\frac{A_{12}+A_{66}}{R}+\frac{2 B_{66}+B_{12}}{R^{2}}, \\
e_{14}=\frac{A_{12}}{R}, \quad e_{15}=-B_{11}, \\
e_{16}=\frac{-B_{12}+2 B_{66}}{R^{2}}, \quad e_{21}=\frac{A_{12}+A_{66}}{R}+\frac{B_{66}+B_{12}}{R^{2}}, \\
e_{22}=A_{66}+\frac{3 B_{66}}{R}+\frac{2 D_{66}}{R^{2}}, \\
e_{24}=\frac{A_{22}}{R^{2}}+\frac{2 B_{22}}{R^{3}}+\frac{D_{22}}{R^{4}}, \\
e_{36}=-\left(\frac{A_{22}}{R^{2}}+\frac{B_{22}}{R^{3}}\right)=-2 B_{66} \\
\left.e_{26}=-\frac{2 D_{66}+D_{12}}{R^{2}}\right), \\
e_{34}=\left(\frac{B_{22}}{R^{3}}+\frac{D_{22}}{R^{4}}\right), \quad e_{31}=\frac{A_{22}}{R^{2}}+\frac{B_{22}}{R^{3}}, \\
e_{32}=-\frac{B_{12}}{R}=-e_{14}, \quad e_{33}=\frac{B_{12}+2 B_{66}}{R^{2}}, \\
R_{66}
\end{gathered}
$$

$$
\begin{gathered}
e_{38}=-\left(\frac{2 D_{12}+4 D_{66}}{R^{2}}\right), \quad e_{39}=-\frac{D_{22}}{R^{4}} \\
e_{310}=\frac{2 B_{12}}{R}, \quad e_{311}=\frac{2 B_{22}}{R^{3}} \\
e_{312}=-\frac{A_{22}}{R^{2}}, \quad e_{313}=\frac{1}{R^{2}}
\end{gathered}
$$

C.

$$
\begin{gathered}
c_{11}=k_{m}^{2} e_{11}+n^{2} e_{12}, \quad c_{12}=i n k_{m} e_{13}, \\
c_{13}=i k_{m}\left(e_{14}-n^{2} e_{16}-k_{m}^{2} e_{15}\right) \\
c_{21}=-i n k_{m} e_{21}, \quad c_{22}=k_{m}^{2} e_{22}+n^{2} e_{23}, \\
c_{23}=-n k_{m}^{2} e_{24}+n e_{25}-n^{3} e_{26}, \\
c_{31}=-i k_{m}\left(e_{14}-n^{2} e_{16}-k_{m}^{2} e_{15}\right) \\
c_{32}=-n k_{m}^{2} e_{24}+n e_{25}-n^{3} e_{26}, \\
c_{33}=e_{312}+n^{2} e_{311}+k_{m}^{2} e_{310}-n^{4} e_{39}-n k_{m}^{2} e_{38}-k_{m}^{4} e_{37} \\
+K+G\left(k_{m}^{2}+e_{313} n^{2}\right) .
\end{gathered}
$$

\section{References}

[1] A. E. H. Love, "On the small free vibrations and deformation of thin elastic Shell," Philosophical Transactions of the Royal Society A, vol. 179, pp. 491-549, 1888.

[2] W. Flügge, Statik und Dynamik der Schalen, Springer-Verlag, Berlin, Germany, 1934.

[3] C. B. Sharma, "Calculation of natural frequencies of fixed-free circular cylindrical shells," Journal of Sound and Vibration, vol. 35, no. 1, pp. 55-76, 1974.

[4] C. B. Sharma, "Vibration characteristics of thin circular cylinders," Journal of Sound and Vibration, vol. 63, no. 4, pp. 581592, 1979.

[5] D. N. Paliwal, R. K. Pandey, and T. Nath, "Free vibrations of circular cylindrical shell on Winkler and Pasternak foundations," International Journal of Pressure Vessels and Piping, vol. 69, no. 1, pp. 79-89, 1996.

[6] C. T. Loy, K. Y. Lam, and J. N. Reddy, "Vibration of functionally graded cylindrical shells," International Journal of Mechanical Sciences, vol. 41, no. 3, pp. 309-324, 1999.

[7] S. C. Pradhan, C. T. Loy, K. Y. Lam, and J. N. Reddy, "Vibration characteristics of functionally graded cylindrical shells under various boundary conditions," Applied Acoustics, vol. 61, no. 1, pp. 111-129, 2000.

[8] X. M. Zhang, G. R. Liu, and K. Y. Lam, "Vibration analysis of thin cylindrical shells using wave propagation approach," Journal of Sound and Vibration, vol. 239, no. 3, pp. 397-403, 2001.

[9] M. M. Najafizadeh and M. R. Isvandzibaei, "Vibration of functionally graded cylindrical shells based on higher order shear deformation plate theory with ring support," Acta Mechanica, vol. 191, no. 1-2, pp. 75-91, 2007. 
[10] S. H. Arshad, M. N. Naeem, and N. Sultana, "Frequency analysis of functionally graded material cylindrical shells with various volume fraction laws," Proceedings of the Institution of Mechanical Engineers C, vol. 221, no. 12, pp. 1483-1495, 2007.

[11] Z. Iqbal, M. N. Naeem, and N. Sultana, "Vibration characteristics of FGM circular cylindrical shells using wave propagation approach," Acta Mechanica, vol. 208, no. 3-4, pp. 237-248, 2009.

[12] S. H. Arshad, M. N. Naeem, N. Sultana, Z. Iqbal, and A. G. Shah, "Vibration of bilayered cylindrical shells with layers of different materials," Journal of Mechanical Science and Technology, vol. 24, no. 3, pp. 805-810, 2010.

[13] A. G. Shah, T. Mahmood, M. N. Naeem, Z. Iqbal, and S. H. Arshad, "Vibrations of functionally graded cylindrical shells based on elastic foundations," Acta Mechanica, vol. 211, no. 3-4, pp. 293-307, 2010.

[14] A. G. Shah, T. Mahmood, and M. N. Naeem, "Vibrations of FGM thin cylindrical shells with exponential volume fraction law," Applied Mathematics and Mechanics, vol. 30, no. 5, pp. 607-615, 2009.

[15] S. H. Arshad, M. N. Naeem, N. Sultana, A. G. Shah, and Z. Iqbal, "Vibration analysis of bi-layered FGM cylindrical shells," Archive of Applied Mechanics, vol. 81, no. 3, pp. 319343, 2011.

[16] M. N. Naeem, M. Ahmad, A. G. Shah, Z. Iqbal, and S. H. Arshad, "Applicability of generalized differential quadrature method for vibration study of fgm cylindrical shells," European Journal of Scientific Research, vol. 47, no. 1, pp. 82-99, 2010.

[17] H. S. Shen, J. Yang, and S. Kitipornchai, "Postbuckling of internal pressure loaded FGM cylindrical shells surrounded by an elastic medium," European Journal of Mechanics, vol. 29, no. 3, pp. 448-460, 2010.

[18] A. H. Sofiyev and H. Avear, "The stability of cylindrical shells containing an FGM Layer subjected to axial load on the Pasternak foundation," Engineering, vol. 2, no. 4, pp. 228-236, 2010.

[19] A. H. Sofiyev, A. N. Alizada, O. Akin, A. Valiyev, M. Avear, and S. Adiguzel, "On the stability of FGM shells subjected to combined loads with different edge conditions and resting on elastic foundations," Acta Mechanica, vol. 223, no. 1, pp. 189204, 2011.

[20] M. N. Naeem and C. B. Sharma, "Prediction of natural frequencies for thin circular cylindrical shells," Proceedings of the Institution of Mechanical Engineers C, vol. 214, no. 10, pp. 1313-1328, 2000. 

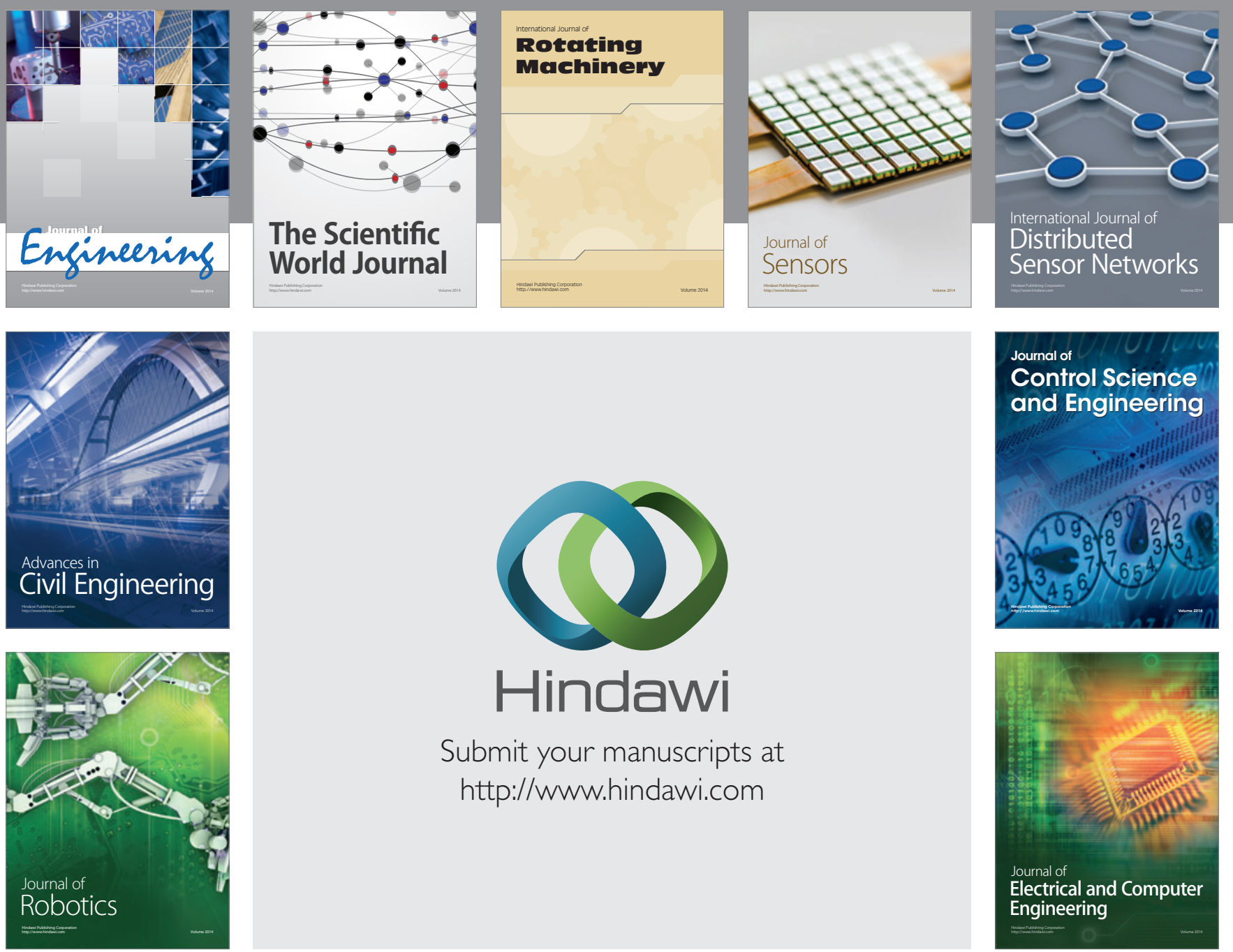

Submit your manuscripts at

http://www.hindawi.com
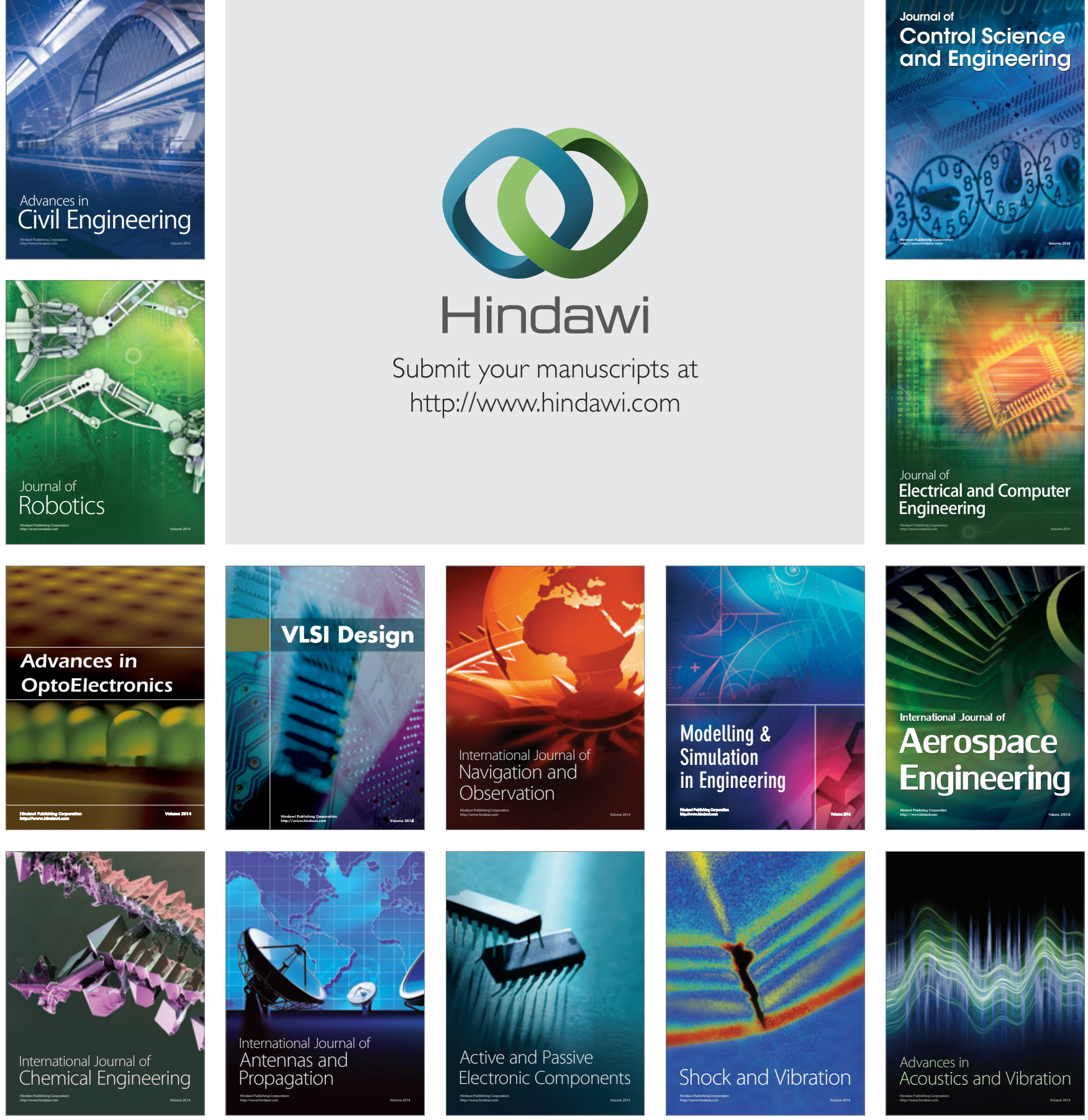